The Astrophysical Journal, 665:936-943, 2007 August 20

(C) 2007. The American Astronomical Society. All rights reserved. Printed in U.S.A.

\title{
A DETAILED STUDY OF GAS AND STAR FORMATION IN A HIGHLY MAGNIFIED LYMAN BREAK GALAXY AT $z=3.07$
}

\author{
K. E. K. Coppin, ${ }^{1}$ A. M. Swinbank, ${ }^{1}$ R. Neri,${ }^{2}$ P. Cox,${ }^{2}$ Ian Smail,,${ }^{1}$ R. S. Ellis,${ }^{3}$ J. E. Geach, ${ }^{1}$ \\ B. Siana, ${ }^{4}$ H. Teplitz, ${ }^{4}$ S. Dye, ${ }^{5}$ J.-P. Kneib ${ }^{6}$ A. C. Edge, ${ }^{1}$ and J. Richard ${ }^{3}$ \\ Received 2007 April 4; accepted 2007 May 10
}

\begin{abstract}
We report the detection of $\mathrm{CO}(3-2)$ emission from a bright, gravitationally lensed Lyman Break galaxy, LBG J213512.73-010143 (the "Cosmic Eye"), at $z=3.07$, using the Plateau de Bure Interferometer. This is only the second detection of molecular gas emission from an LBG and yields an intrinsic molecular gas mass of $(2.4 \pm 0.4) \times 10^{9} M_{\odot}$. The lens reconstruction of the UV morphology of the LBG indicates that it comprises two components separated by $\sim 2 \mathrm{kpc}$. The CO emission is unresolved, $\theta \lesssim 3^{\prime \prime}$, and appears to be centered on the intrinsically fainter (and also less highly magnified) of the two UV components. The width of the CO line indicates a dynamical mass of $(8 \pm 2) \times 10^{9} \csc ^{2} i M_{\odot}$ within the central $2 \mathrm{kpc}$. Employing mid-infrared observations from Spitzer, we infer a stellar mass of $M_{*} \sim(6 \pm 2) \times$ $10^{9} M_{\odot}$ and a star formation rate of $\sim 60 M_{\odot} \mathrm{yr}^{-1}$, indicating that the molecular gas will be consumed in $\lesssim 40 \mathrm{Myr}$. The gas fractions, star formation efficiencies, and line widths suggests that LBG J213512 is a high-redshift, gas-rich analog of a local luminous infrared galaxy. This galaxy has a similar gas-to-dynamical mass fraction as observed in the submillimeterselected population, although the gas surface density and star formation efficiency is a factor of 3 times less, suggesting less vigorous activity. We discuss the uncertainties in our conclusions arising from adopting a CO-to- $\mathrm{H}_{2}$ conversion factor appropriate for either the Milky Way or local luminous infrared galaxies. These observations demonstrate that current facilities, when aided by fortuitous gravitational magnification, can study "ordinary" galaxies at high redshift and so act as pathfinders for ALMA.
\end{abstract}

Subject headings: cosmology: observations — galaxies: evolution — galaxies: formation — galaxies: individual (LBG J213512.73-010143) — galaxies: kinematics and dynamics — galaxies: starburst

\section{INTRODUCTION}

Lyman Break galaxies (LBGs) were the first significant population of high-redshift galaxies to be identified (Steidel et al. 1996). Subsequent work has shown that they represent only a subset of the galaxy population at $z \sim 3$, whose properties in part reflect their selection: LBGs are actively star-forming galaxies with relatively low dust obscuration. However, LBGs are still the most common population at this epoch, and as such they have been interpreted as a phase in the formation of "typical" galaxies (Somerville et al. 2001; Baugh et al. 2005). Thus, understanding their properties, such as the distribution of star formation and dynamical, stellar, and gas masses, may be a critical element in constraining models for the formation and evolution of normal galaxies.

Studies of the physical properties of LBGs have so far traced their star formation rates and histories, stellar and dynamical masses, and morphologies (e.g., Shapley et al. 2001, 2003, 2005; Reddy \& Steidel 2004; Law et al. 2007). These studies have motivated more detailed investigations of the properties of LBGs, in particular to determine the gas content of these galaxies and the chemical enrichment of this gas, to more fully understand their evolutionary status. In particular, the gas content of these galaxies (as traced

\footnotetext{
${ }^{1}$ Institute for Computational Cosmology, Durham University, South Road, Durham, DH13LE, UK; kristen.coppin@dur.ac.uk.

${ }^{2}$ Institut de Radioastronomie Millimétrique (IRAM), 300 rue de la Piscine, Domaine Universitaire, 38406 Saint Martin d'Hères, France.

${ }^{3}$ Caltech, MC 105-24, 1200 East California Boulevard, Pasadena, California, CA 91125.

${ }^{4}$ Spitzer Science Center, Caltech, MC 314-6, 1200 East California Boulevard, Pasadena, California, CA 91125.

${ }^{5}$ School of Physics and Astronomy, Cardiff University, 5, The Parade, Cardiff, Wales, CF24AA, UK.

${ }^{6}$ Laboratoire d'Astrophysique de Marseille, Traverse du Siphon-B.P.8 13376, Marseille Cedec 12, France.
}

by their $\mathrm{CO}$ emission in the millimeter wave band) is a key observable, as this cold and dense gas provides the reservoir for star formation activity (and hence the potential to build up a substantial stellar mass). CO emission provides both a reliable measure of the gas mass and also an unbiased tracer of its dynamics and hence the mass of the host galaxy.

However, the properties of the gaseous component in typical LBGs have proved hard to address, as they are beyond the sensitivity limits of the relevant current observational facilities. Progress has so far only been made through studies of a single, rare example of an LBG whose apparent brightness is boosted by gravitational magnification by a foreground cluster lens: MS 1512-cB58 (hereafter referred to as cB58: Yee et al. 1996). LBG cB58 has been the subject of two unique studies of the interstellar medium of LBGs: Pettini et al. $(2000,2002)$ derived the elemental abundances from high signal-to-noise $(\mathrm{S} / \mathrm{N})$, high-resolution rest-frame UV spectroscopy, concluding that $\mathrm{cB} 58$ is a $Z \sim 0.5 Z_{\odot}$ starburst galaxy, while Baker et al. (2004) obtained for cB58 the only detection of $\mathrm{CO}$ emission from an LBG, providing the first direct evidence of the existence of a sizeable cold gas reservoir in an LBG. These observations have also helped to shed light on several key details of the star formation process in this young galaxy.

Unfortunately, while these studies of cB58 have provided unique insights into the properties of LBGs, it is dangerous to draw general conclusions about the whole LBG population from this single example. Hence, significant efforts have gone into finding other examples of lensed LBGs, and after nearly a decade of searches, several lensed LBGs as bright as cB58 have now been found. Smail et al. (2007) present the discovery of a strongly lensed LBG at $z=3.07$, LBG J213512.73-010143 (hereafter LBG J213512), similar to cB58. At $r_{\mathrm{AB}}=20.3$, this new LBG is brighter in the rest-frame UV than cB58, owing to the $28 \times$ magnification (Dye et al. 2007). Moreover, the LBG appears as two 


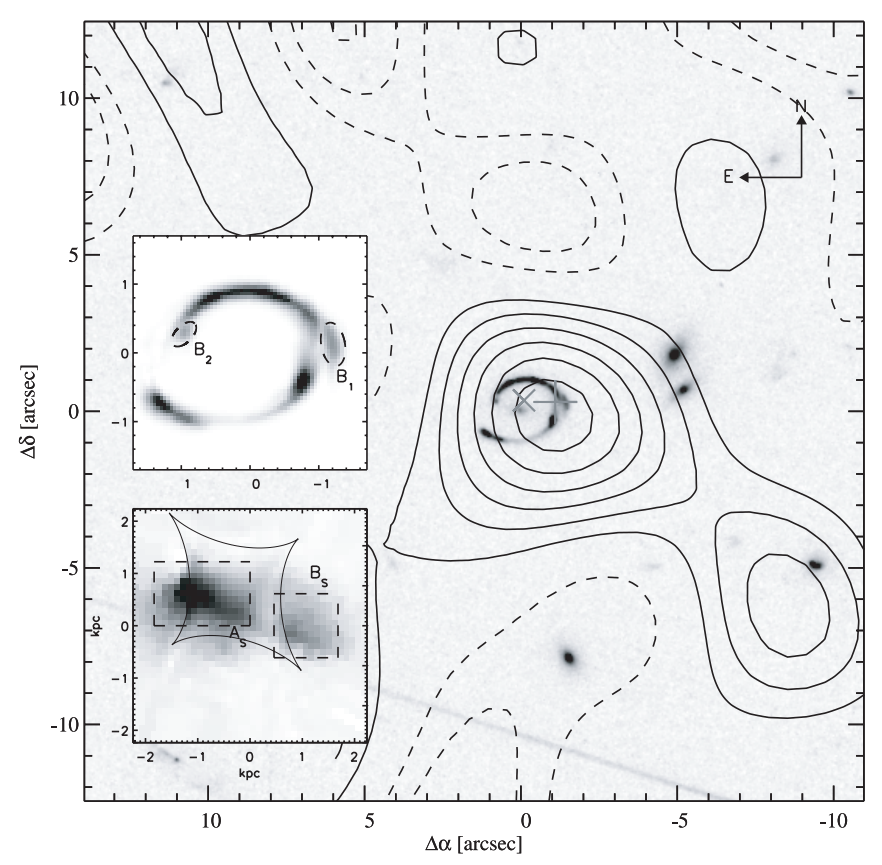

FIG. 1.- HST ACS F606W image of LBG J213512, centered on the optical position $21^{\mathrm{h}} 35^{\mathrm{m}} 12.73^{\mathrm{s}},-01^{\circ} 01^{\prime} 43.0^{\prime \prime}(\mathrm{J} 2000.0)$, with the $\mathrm{CO}(3-2)$ contours overlaid (contours begin at $1 \sigma$ and increase in steps of $1 \sigma$; negative contours are dashed) We also show two insets, the upper inset illustrates the image-plane morphology of the LBG (with the foreground lens removed; scale in arcseconds) consisting of two bright arcs and two fainter knots (marked $\mathrm{B}_{1}$ and $\mathrm{B}_{2}$ ). The lower inset illustrates the sourceplane reconstruction of the LBG from Dye et al. (2007) in kpc. This shows that in the rest-frame $U V$, the $L B G$ comprises two components $\left(\mathrm{A}_{\mathrm{s}}\right.$ and $\left.\mathrm{B}_{\mathrm{s}}\right)$ separated by $\sim 2-$ $3 \mathrm{kpc}$. Both of the bright arcs are formed from the lensing of $A_{s}$, which is magnified in total by a factor of $28 \times$, while $B_{s}$ gives rise to $B_{1}$ and $B_{2}$ (the magnifications of $B_{1}$ and $B_{2}$ are $6.2 \times$ and $1.8 \times$, respectively). Comparison of the $C O$ map with the optical morphology of the system shows that the $\mathrm{CO}$ emission is offset from the center of the LBG, and we mark by a cross the predicted center of the $\mathrm{CO}$ emission if the molecular gas follows the $R$ - or $K$-band light. We conclude that the $\mathrm{CO}$ emission is unlikely to follow the rest-frame UV/optical light in this system, and instead it appears to coincide with a faint knot (marked as $\mathrm{B}_{1}$ in the top inset). To test this, we predict the observed centroid of the $\mathrm{CO}$ emission if it is associated with source $\mathrm{B}_{\mathrm{s}}$ and mark this as a plus sign; this is coincident with the observed centroid supporting the proposed association. See text for a detailed discussion.

small $\operatorname{arcs}\left(\sim 3^{\prime \prime}\right.$ in extent) and hence provides a unique opportunity for spatially resolved studies on $100 \mathrm{pc}$ scales across this galaxy. When corrected for the lensing magnification, the background source appears as an $L^{\star} \mathrm{LBG}$, with a similar intrinsic luminosity to $\mathrm{cB} 58$. We thus have a second example of a highly magnified "typical" LBG.

In this paper, we present interferometric measurements of the $\mathrm{CO}(3-2)$ emission and mid-infrared photometric observations of this new lensed LBG. We use the $\mathrm{CO}(3-2)$ line luminosity and width to infer the gas and dynamical masses and compare these with the stellar mass inferred from the rest-frame optical-to-nearinfrared photometry of the system. We also compare our results with the similar observations of $\mathrm{cB} 58$ to investigate the variations within the LBG population and further compare our results with the growing number of $\mathrm{CO}$ observations of other high-redshift galaxies (see Solomon \& Vanden Bout 2005 for a review). We adopt cosmological parameters from the $W M A P$ fits in Spergel et al. (2003): $\Omega_{\Lambda}=0.73, \Omega_{m}=0.27$, and $H_{0}=71 \mathrm{~km} \mathrm{~s}^{-1} \mathrm{Mpc}^{-1}$. All quoted magnitudes are on the $\mathrm{AB}$ system.

\section{OBSERVATIONS AND REDUCTION}

\subsection{Millimeter Interferometry}

We used the six-element IRAM PdBI (Guilloteau et al. 1992) to observe LBG J213512 in the redshifted $\mathrm{CO}(3-2)$ line and in

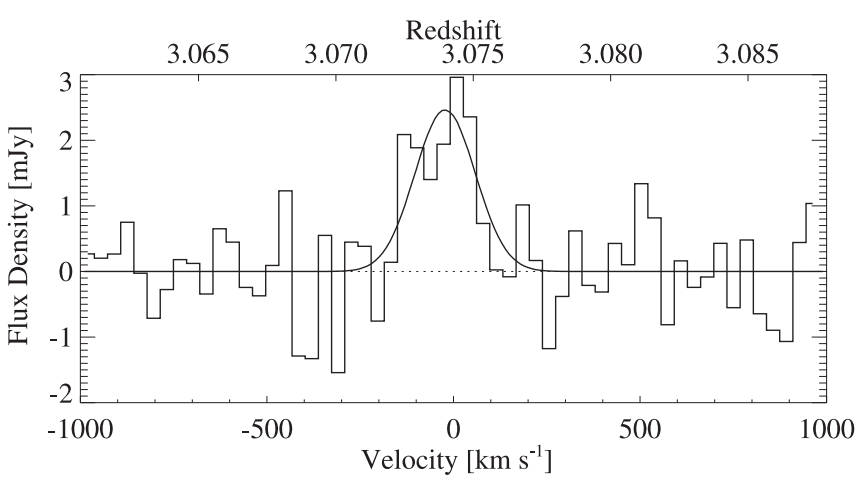

FIG. 2.-Spectrum of $\mathrm{CO}(3-2)$ emission in LBG J213512, binned into $35.3 \mathrm{~km} \mathrm{~s}^{-1}$ channels. We detect the line at $7.1 \sigma$ significance, and the best-fitting Gaussian with a FWHM of $190 \pm 24 \mathrm{~km} \mathrm{~s}^{-1}$ is overlaid. The upper axis indicates the redshift of the $\mathrm{CO}(3-2)$ transition. There is a hint of double peak to the $\mathrm{CO}$ line, although a double-component fit does not provide a better description of the data, due to the modest $\mathrm{S} / \mathrm{N}$ of the detection. We have confirmed that there is no detectable spatial offset between the blue and red halves of the line $\left(\lesssim 0.1^{\prime \prime}\right)$.

the continuum at $84.87 \mathrm{GHz}$. The frequency was tuned to the $\mathrm{CO}(3-2)$ rotational transition at $z=3.0743$, the systemic redshift of the system from Smail et al. (2007). Observations were made in D configuration in Director's Discretionary Time (DDT) between 2006 August 24 and 2006 September 20 with good atmospheric phase stability (seeing $=0.6^{\prime \prime}-1.6^{\prime \prime}$ ) and reasonable transparency ( $\mathrm{pwv}=5-15 \mathrm{~mm}$ ). We observed LBG J213512 with a total on-source observing time of $10 \mathrm{hr}$. The spectral correlator was adjusted to detect the line with a frequency resolution of $2.5 \mathrm{MHz}$ in the $580 \mathrm{MHz}$ band of the receivers. The overall flux scale for each observing epoch was set on 2134+004, 2145+ 067 , and MWC 349. The visibilities were resampled to a velocity resolution of $35.3 \mathrm{~km} \mathrm{~s}^{-1}(10 \mathrm{MHz})$, providing $1 \sigma$ line sensitivities of $\sim 1.0 \mathrm{mJy}$ beam $^{-1}$. The corresponding synthesized beam, adopting natural weighting, was $6.3^{\prime \prime} \times 5.5^{\prime \prime}$ at $44^{\circ}$ east of north. The data were calibrated, mapped, and analyzed in the gildas software package. Inspection of the velocity data cube shows a significant detection of $\mathrm{CO}(3-2)$ line emission close to the position of LBG J213512 in the central velocity channels with a velocity width of $\sim 250 \mathrm{~km} \mathrm{~s}^{-1}$. Figure 1 shows a channel map constructed from the average emission in a $250 \mathrm{~km} \mathrm{~s}^{-1}$ window centered on the systemic redshift of the system. In Figure 2, we show the spectrum of the $\mathrm{CO}(3-2)$ emission in the brightest pixel of the source in the channel map.

\subsection{Mid-Infrared Imaging}

Observations of LBG J213512 were taken with the Spitzer Space Telescope Infrared Array Camera (IRAC) in 2005 November using time awarded through DDT. Observations were taken at $3.6,4.5,5.8$, and $8.0 \mu \mathrm{m}$ using two cycles of a 10 point dither pattern, each with an integration time of $30 \mathrm{~s}$. The total integration time was $0.9 \mathrm{ks} \mathrm{pixel}^{-1}$ in each band. Further observations were also obtained at $24 \mu \mathrm{m}$ with the Multiband Imaging Photometer for Spitzer (MIPS) camera. These observations were made using eight repeats of a nine-point dither pattern, each consisting of $30 \mathrm{~s}$ exposures, for a total integration time of $2.0 \mathrm{ks}$. We use the Post Basic Calibrated IRAC frames generated by the pipelines at the Spitzer Science Center (SSC) and perform postprocessing on the data frames to remove common artifacts and flatten smalland large-scale gradients using "master flats" generated from the data. For mosaicking, we use the SSC mopex package, which makes use of the supplementary calibration files supplied with the main science set. We align and combine the four IRAC images and use this composite image to extract 3.8" diameter aperture 
TABLE 1

Aperture-Corrected Photometry for LBG J213512

\begin{tabular}{|c|c|c|c|}
\hline $\begin{array}{l}\text { Band } \\
\text { (1) }\end{array}$ & $\begin{array}{l}\mathrm{LBG}+\text { Lens } \\
\text { (2) }\end{array}$ & $\begin{array}{l}\text { Lens } \\
\text { (3) }\end{array}$ & $\begin{array}{l}\text { LBG } \\
\text { (4) }\end{array}$ \\
\hline$g$ & $\ldots$ & & $21.47[5]$ \\
\hline$R_{606} \ldots \ldots \ldots \ldots$ & $\ldots$ & $22.34[15]$ & $20.54[2]$ \\
\hline K & $\ldots$ & $19.70[10]$ & $18.90[10]$ \\
\hline $3.6 \mu \mathrm{m}$ & $18.34[2]$ & $\ldots$ & $18.74[4]$ \\
\hline $4.5 \mu \mathrm{m}$ & $18.19[2]$ & $\ldots$ & $18.39[4]$ \\
\hline $5.8 \mu \mathrm{m}$ & $18.13[3]$ & $\ldots$ & $18.33[6]$ \\
\hline $8.0 \mu \mathrm{m}$ & $18.30[2]$ & $\ldots$ & $18.35[4]$ \\
\hline $24 \mu \mathrm{m}$ & $17.78[25]$ & $\ldots$ & $17.78[25]$ \\
\hline
\end{tabular}

Notes.-Col. (2): The observed photometry for the LBG. Due to the low spatial resolution of IRAC, the photometry from the $z=0.73$ and $z=3.07$ galaxies are blended. We remove the contribution of the $z=0.73$ lensing galaxy to the IRAC channels by fitting the $R_{606}$ and $K$-band photometry of the foreground lens (col. [3]) with an elliptical galaxy template redshifted to $z=0.73$ and subtracting the contribution at $\geq 3.6 \mu \mathrm{m}$. These corrected LBG photometry are given in col. (4) (see text). To convert to intrinsic magnitudes (corrected for the lensing amplification factor $28 \pm 3 \times$ ), add $3.6 \mathrm{mag}$ to col. (4). Values in brackets denote the error in the last decimal place, and we note that $1 \mu \mathrm{Jy}$ corresponds to $m_{\mathrm{AB}}=23.90$.

magnitudes at the position of LBG J213512 from the IRAC data and $10^{\prime \prime}$ diameter aperture magnitudes from the MIPS data. We obtain a strong detection of the emission from the LBG J213512 (with some contribution from the foreground lens) at 3.6-8 $\mu \mathrm{m}$ and a weaker detection at $24 \mu \mathrm{m}$. Aperture-corrected photometry for the LBG are reported in Table 1 , including $g-R_{606^{-}}$, and $K$-band photometry from Smail et al. (2007).

\section{ANALYSIS AND RESULTS}

\subsection{CO Emission Properties}

The luminosity, velocity width, and spatial extent of the CO line emission can be used to place limits on the gas and dynamical mass of the system. Fitting a Gaussian profile to the $\mathrm{CO}$ spectrum for the source at the phase center of our observations (Fig. 2), we derive a best-fit redshift for the $\mathrm{CO}(3-2)$ emission of $z=$ $3.0740 \pm 0.0002$. This is in excellent agreement with the systemic redshift derived from the [O III] $\lambda 5007$ line by Smail et al. (2007), with an offset of just $\Delta v=-22 \pm 14 \mathrm{~km} \mathrm{~s}^{-1}$. The error on the line parameters are bootstrap estimates generated by adding noise at random to the channels closest to the spectral peak from the outermost noisy baseline channels 1000 times with replacement (e.g., see Wall \& Jenkins 2003). This provides a reasonable estimate of the uncertainties, provided the noise is not strongly correlated between channels. The rest-frame FWHM of the CO line is $190 \pm 24 \mathrm{~km} \mathrm{~s}^{-1}$ (or alternatively a dispersion of $80 \pm 10 \mathrm{~km} \mathrm{~s}^{-1}$ ), which is consistent with the $[\mathrm{O}$ III $] 25007$ emission line (FWHM $\lesssim 220 \mathrm{~km} \mathrm{~s}^{-1}$ ), as measured by Smail et al. (2007). The velocity integrated line flux is measured to be $F_{\mathrm{CO}(3-2)}=$ $0.50 \pm 0.07 \mathrm{Jy} \mathrm{km} \mathrm{s}^{-1}(\mathrm{~S} / \mathrm{N}=7.1)$. We note that this line flux is comparable to the $\mathrm{CO}$ flux density measured for $\mathrm{CB} 58: F_{\mathrm{CO}(3-2)}=$ $0.37 \pm 0.08 \mathrm{Jy} \mathrm{km} \mathrm{s}^{-1}$ (Baker et al. 2004, with an estimated magnification factor for $\mathrm{cB} 58$ of $\mu \sim 32$ ).

Next, averaging the emission over a $250 \mathrm{~km} \mathrm{~s}^{-1}$ window centered on the redshift of the $\mathrm{CO}$ emission, we determine a flux of $2.0 \pm 0.3 \mathrm{mJy}_{\text {beam }}{ }^{-1}$ for a source centered at $21^{\mathrm{h}} 35^{\mathrm{m}} 12.62^{\mathrm{s}}$, $-01^{\circ} 01^{\prime} 43.9^{\prime \prime}$ (J2000.0), corresponding to a $\sim 7 \sigma$ detection (we ignore the small correction for primary beam attenuation, given that the source is close to the phase center of the map). We note that no significant continuum emission is detected from the linefree region ( $470 \mathrm{MHz}$ of bandwidth) down to a $1 \sigma$ sensitivity of $0.14 \mathrm{mJy}$.
In Figure 1, we show the Hubble Space Telescope (HST) ACS F606W image of the system from Smail et al. (2007) and overlay the contours from the $\mathrm{CO}(3-2)$ channel map. Dye et al. (2007) present a detailed lens model and use it to reconstruct the restframe UV source-plane morphology of the LBG. They show that the system comprises two UV-emitting components separated by $\sim 2-3 \mathrm{kpc}$, probably representing two star-forming regions within the galaxy separated by a dust lane or region of lower activity (see inset in Fig. 1; $A_{s}$ and $B_{s}$ ). The intrinsically brighter and more highly amplified of these, $A_{s}$, lies within the lens caustic and is magnified by a factor of $28 \pm 3$ (Smail et al. 2007), giving rise to the ringlike structure surrounding the foreground galaxy. The second $\mathrm{UV}$ component, $\mathrm{B}_{\mathrm{s}}$, lies just outside the caustic and gives rise to two images: $B_{1}$ and $B_{2}$ (see Fig. 1; also see Fig. 4 of Dye et al. 2007). The lensing magnifications of the components are $6.2 \times$ and $1.8 \times$, respectively, giving a total amplification factor of $(8.0 \pm 0.9) \times$ for $\mathrm{B}_{\mathrm{s}}$ (Dye et al. 2007).

The astrometric solution on the HST image is derived by comparing the positions of unsaturated stars with the USNOA-2.0 catalog, and we estimate an rms uncertainty in the optical position of the system $\left(21^{\mathrm{h}} 35^{\mathrm{m}} 12.73^{\mathrm{s}},-01^{\circ} 01^{\prime} 43.0^{\prime \prime}\right.$ [J2000.0]) of $\lesssim 0.2^{\prime \prime}$. The absolute astrometry of the $\mathrm{CO}$ observations is estimated to be accurate to $\lesssim 0.01^{\prime \prime}$, but the centroid for the emission is only accurate to $\lesssim 0.3^{\prime \prime}$ and places the peak of the $\mathrm{CO}$ emission at $21^{\mathrm{h}} 35^{\mathrm{m}} 12.62^{\mathrm{s}},-01^{\circ} 01^{\prime} 43.9^{\prime \prime}(\mathrm{J} 2000.0)$, which is centered approximately $1.9^{\prime \prime} \pm 0.4^{\prime \prime}$ due west of the optical centroid.

We first test whether the CO centroid is consistent with the expected position if the $\mathrm{CO}$ emission traces the rest-frame $\mathrm{UV} /$ optical light. To achieve this, we subtract the foreground lensing galaxy from the HST imaging and convolve the resulting image with the PdBI beam; this predicts a centroid for the $\mathrm{CO}$ emission approximately $0.4^{\prime \prime} \mathrm{NW}$ of the lensing galaxy (marked by the cross in Fig. 1). Thus, the CO line emission does not appear to be associated with the most highly magnified and UV-bright component part of the galaxy $\left(A_{s}\right)$. Instead, the centroid of the $C O$ emission appears to correspond to $\mathrm{B}_{1}$, the more magnified of the two images of component $\mathrm{B}_{\mathrm{s}}$. To confirm this, we model the $\mathrm{CO}$ emission from $B_{s}$ using the magnifications for the two images from Dye et al. (2007) and convolve this with the PdBI beam; this yields a centroid of $21^{\mathrm{h}} 35^{\mathrm{m}} 12.63^{\mathrm{s}},-01^{\circ} 01^{\prime} 43.3^{\prime \prime}(\mathrm{J} 2000.0$; marked by the plus sign in Fig. 1), which is $0.6^{\prime \prime} \pm 0.4^{\prime \prime}$ due west from the observed peak and hence consistent with it. Thus, we conclude that the $\mathrm{CO}$ reservoir appears to be associated with $\mathrm{B}_{\mathrm{s}}$, the fainter of the two UV regions in LBG J213512. We discuss the implications of this further in $\S 4$.

\subsection{CO Luminosity and Molecular Gas Mass}

We calculate the line luminosity and estimate the total cold gas mass $\left(\mathrm{H}_{2}+\mathrm{He}\right)$ from the integrated $\mathrm{CO}$ line flux. The observed $\mathrm{CO}(3-2)$ line luminosity is $\mu L_{\mathrm{CO}(3-2)}^{\prime}=(2.4 \pm 0.3) \times$ $10^{10} \mathrm{~K} \mathrm{~km} \mathrm{~s}^{-1} \mathrm{pc}^{2}$, where $\mu$ is the amplification factor. Although the lensing magnification of the entire UV source is $\sim 28 \times$, our association of the molecular gas reservoir with component $B_{s}$ means we must adopt the estimated magnification for this component of $(8.0 \pm 0.9) \times$. The resulting intrinsic line luminosity is therefore $L_{\mathrm{CO}(3-2)}^{\prime}=(3.0 \pm 0.5) \times 10^{9} \mathrm{~K} \mathrm{~km} \mathrm{~s}^{-1} \mathrm{pc}^{2}$. We then assume both a line luminosity ratio of $r_{32}=L_{\mathrm{CO}(3-2)}^{\prime} / L_{\mathrm{CO}(1-0)}^{\prime}=1$ (i.e., a constant brightness temperature) and a CO-to- $\mathrm{H}_{2}$ conversion factor of $\alpha=0.8 M_{\odot}\left(\mathrm{K} \mathrm{km} \mathrm{s}^{-1} \mathrm{pc}^{2}\right)^{-1}$. These values are appropriate for local galaxy populations exhibiting levels of star formation activity similar to LBG J213512 (e.g., local infrared galaxies [LIRGs]; Solomon et al. 1997; see § 3.4). We discuss in $\S 4.2$ how our conclusions would change if we adopted $\alpha$ and $r_{32}$ values typical of the Milky Way. This then yields a total cold gas 
mass of $M_{\text {gas }}=M\left(\mathrm{H}_{2}+\mathrm{He}\right)=\alpha L_{\mathrm{CO}}^{\prime}=(2.4 \pm 0.4) \times 10^{9} M_{\odot}$. Based on the association of the $\mathrm{CO}$ emission with source $\mathrm{B}_{\mathrm{s}}$ and its extent in the rest-frame UV, we assume the gas is distributed in a disk with a radius no larger than $1 \mathrm{kpc}$ (see Fig. 1), resulting in an inferred gas surface density of $\Sigma_{\text {gas }} \simeq(760 \pm 130) M_{\odot} \mathrm{pc}^{-2}$.

\subsection{Dynamical and Stellar Mass}

The next step in our analysis is to compare the mass we derived for the cold gas reservoir to the dynamical and stellar masses in this LBG. While in principle the dynamical masses of LBGs can be derived from optical or near-infrared observations of emissionline gas in these galaxies, dust obscuration and outflows in the gas may bias the measurements. In contrast, molecular $\mathrm{CO}$ emission is comparatively immune to the effects of obscuration and outflows and therefore provides an unbiased measurement of dynamics within the $\mathrm{CO}$ emitting region. Our $\mathrm{CO}$ observations allow us to place strong constraints on the dynamical mass, while the Spitzer observations can be used to constrain the stellar mass and current star formation rate (SFR) of the galaxy.

For LBG J213512, the line width of the CO emission (190 \pm $\left.24 \mathrm{~km} \mathrm{~s}^{-1}\right)$ predicts a dynamical mass of $(8.4 \pm 1.4) \times 10^{9} \csc ^{2} i M_{\odot}$, assuming the gas lies in a disk with inclination $i$ and a radius of $\sim 1 \mathrm{kpc}$. Based on this, we calculate a gas-to-dynamical mass fraction of $f=M_{\mathrm{gas}} / M_{\mathrm{dyn}} \sim 0.30 \sin ^{2} i$. For comparison with the total stellar mass, we extrapolate this $\mathrm{CO}$ measurement slightly to give us a total mass within the UV extent of the $\mathrm{LBG}$, a radius of $2 \mathrm{kpc}$, yielding an enclosed mass of $M(<2 \mathrm{kpc}) \sim 1.7 \times 10^{10} \csc ^{2} i M_{\odot}$. We note that the mean angle of randomly oriented disks with respect to the sky plane in three dimensions is $i=30^{\circ}$ (Carilli \& Wang 2006), resulting in an inclination correction of $\csc ^{2} i=4$.

In order to derive the stellar mass, we adopt the approach of Borys et al. (2005): first we estimate the rest-frame $K$-band luminosity for the galaxy by interpolating the rest-frame spectral energy distribution (SED) and then combine this with estimates of the $K$-band light-to-mass ratio $\left(L_{K} / M\right)$ for a range of plausible ages for the dominant stellar population. We exploit the HyperZ package (Bolzonella et al. 2000) to fit the observed $g, R_{606}, K$, and IRAC $3.6-8.0 \mu \mathrm{m}$ photometry in Table 1 . We use solar metallicity stellar population models from Bruzual \& Charlot (1993) and a Calzetti et al. (2000) starburst attenuation to infer the rest-frame optical/near-infrared SED. At $z=3.07$, the restframe $K$ band (which is most sensitive to the underlying stellar population) is redshifted to $\sim 8 \mu \mathrm{m}$ (i.e., into IRAC Channel 4 ). However, since the IRAC photometry has a spatial resolution of $\sim 3^{\prime \prime}$, comparable to the extent of the lensed LBG, the contribution from the $z=0.73$ lensing galaxy is blended with the LBG, and the lensing galaxy must be subtracted. We therefore fit the $R_{606^{-}}$and $K$-band magnitudes of the lens with an early-type (E/S0) galaxy template based on the spectral properties and colors in Smail et al. (2007; the template comprises a stellar population for an exponential star formation history with a timescale of $1 \mathrm{Gyr}$ ) redshifted to $z=0.73$ and subtract the expected contribution to the IRAC photometry: $\$ 20 \%, 15 \%, 10 \%$, and $5 \%$ at $3.6,4.5,5.0$, and $8.0 \mu \mathrm{m}$, respectively. The LBG photometry with the contribution from the lensing galaxy removed is given in Table 1.

We determine a rest-frame absolute $K$-band magnitude of $m_{K}=-22.2 \pm 0.1$ (corrected for lens magnification using $\mu=$ 28). As expected, this is comparable to $L_{8 \mu \mathrm{m}}^{*}$ for $z \sim 3$ LBGs (Shapley et al. 2005). To convert this to a stellar mass, we need to determine $L_{K} / M$ for the dominant stellar population. To do this, we turn to the Starburst 99 stellar population model (Leitherer et al. 1999), which provides estimates for $L_{K} / M$ for models of bursts of star formation. We adopt an age for the stellar popu- lation dominating the UV light of $\sim 10-30 \mathrm{Myr}$, based on the analysis of the features in the rest-frame UV spectrum which show that the UV emission is dominated by B stars (Smail et al. 2007). Fits using HyperZ (Bolzonella et al. 2000) to the broadband SED spanning the rest-frame $0.13-2 \mu \mathrm{m}$ yield similarly young ages, $\lesssim 50 \mathrm{Myr}$ with moderate reddening, $A_{V} \sim 1$, as do more sophisticated modeling (Shapley et al. 2005). For these ages, the Starburst 99 models predict $L_{K} / M \sim 2.5 \pm 0.5$. We note that the average $L_{K} / M$ for LBGs in Shapley et al. (2005) is $L_{K} / M \sim 2.5$. Thus, for a stellar population dominated by young stars $(<50 \mathrm{Myr})$, the predicted stellar mass is $\sim(6 \pm 2) \times 10^{9} M_{\odot}$. This implies a baryonic mass of $M_{\text {bary }}=M_{\text {gas }}+M_{\text {stars }} \lesssim 1 \times 10^{10} M_{\odot}$, with $75 \%$ of this in stars. This baryonic mass estimate is consistent with the dynamical mass with a $1-2 \mathrm{kpc}$ radius traced by the $\mathrm{CO}$ emission: $M \sim 0.8-1.7 \times 10^{10} \csc ^{2} i M_{\odot}$, for all inclinations.

\subsection{Star Formation Rate and Efficiency}

Smail et al. (2007) estimate the SFR for LBG J213512 from the rest-frame $1500 \AA$ continuum flux of $L_{1500} \sim 4.6 \times 10^{30} \mathrm{erg} \mathrm{s}^{-1}$ $\mathrm{Hz}^{-1}$, which translates into a SFR (corrected for lens magnification and dust reddening) of $\sim 100 M_{\odot} \mathrm{yr}^{-1}$ (assuming a Salpeter initial mass function [IMF] with an upper mass cut off of $100 M_{\odot}$; Kennicutt 1998). Perhaps more reliably, the $24 \mu \mathrm{m}$ flux of the LBG can be used to estimate the far-infrared luminosity of the galaxy (e.g., Bell et al. 2005; Geach et al. 2006), which can then be converted into a SFR. Taking the library of dusty, starforming SEDs from Dale \& Helou (2002), we calculate the ratio of the $24 \mu \mathrm{m}$ luminosity to the far-infrared luminosity integrated over the entire SED $(8-1000 \mu \mathrm{m})$. The SED templates are simply characterized by a power-law distribution of dust mass, with an exponent of 1-2.5 for "typical" local star-forming galaxies (Dale et al. 2001). We derive $L_{\mathrm{FIR}} \sim 3.4 \times 10^{11} L_{\odot}$ for an unlensed $24 \mu \mathrm{m}$ flux of $10 \mu \mathrm{Jy}$, corresponding to a SFR of $\sim 60 M_{\odot} \mathrm{yr}^{-1}$ (Kennicutt 1998). This is the average luminosity over the entire library of SEDs, and the extreme SEDs suggest our estimate is likely to be uncertain by a factor of 3 . This bolometric luminosity is consistent with the $0.14 \mathrm{mJy}$ upper limit to the $3 \mathrm{~mm}$ flux of LBG J213512, and the luminosity implies an $850 \mu \mathrm{m}$ flux of $\sim 0.35 \mathrm{mJy}$, where we have estimated this flux by scaling a modified blackbody with a dust temperature of $40 \mathrm{~K}$ and a dust emissivity $\beta=1.5$ (see Blain et al. 2002) to match the $24 \mu \mathrm{m}$ predicted bolometric luminosity. Note that $S_{850} \sim 0.35 \mathrm{mJy}$ is consistent with a mean observed $850 \mu \mathrm{m}$ flux of $0.5 \pm 0.4 \mathrm{mJy}$ for typical LBGs (Chapman et al. 2000). Our SFR estimate yields a SFR density of $\Sigma_{\mathrm{SFR}} \sim 20 M_{\odot} \mathrm{yr}^{-1} \mathrm{kpc}^{-2}$ for a disk radius of $1 \mathrm{kpc}$, and a star formation efficiency (SFE) of $L_{\mathrm{FIR}} / M_{\mathrm{H}_{2}}=3.4 \times$ $10^{11} L_{\odot} / 2.4 \times 10^{9} M_{\odot} \sim 140 L_{\odot} M_{\odot}^{-1}$.

A related way to present these observations is to roughly estimate the gas-to-dust mass ratio. Following Blain et al. (2002), we estimate a dust mass of $M_{\text {dust }} \simeq 2.5 \times 10^{7} M_{\odot}$ from the predicted $850 \mu \mathrm{m}$ flux of $\sim 0.35 \mathrm{mJy}$, assuming a dust mass absorption coefficient of $\kappa_{850 \mu \mathrm{m}}=0.15 \mathrm{~m}^{2} \mathrm{~kg}^{-1}$. This yields a constraint on the gas-to-dust mass ratio for LBG J213512 of $\sim 100$, with at least a factor of $\sim 6$ uncertainty accounting for our uncertainty of the dust temperature $\left(\Delta T_{\text {dust }} \simeq \pm 5 \mathrm{~K}\right)$, dust emissivity coefficient $(\Delta \beta \simeq \pm 0.5$ ), and mass absorption coefficient (a factor of $\sim 3$; e.g., Seaquist et al. 2004).

In terms of the spatial distribution of the star formation, if the $24 \mu \mathrm{m}$ emission traces the $R$-band light, then we expect that the UV-bright component $A_{s}$ will contain $75 \%$ of the current star formation. Given the poor spatial resolution of our $24 \mu \mathrm{m}$ image and its modest $\mathrm{S} / \mathrm{N}$, it is impossible to determine if the emission follows the UV or CO morphology of the source. However, we have confirmed that within the large uncertainty, the integrated 
$R / 24 \mu \mathrm{m}$ color of the LBG is similar to that seen in other $z=$ 3 LBGs (Reddy et al. 2006), supporting the assumption that the $24 \mu \mathrm{m}$ follows the $R$-band light.

Assuming that the molecular gas reservoir we detect is fueling the star formation within this galaxy, then it has enough gas to sustain the current star formation for

$\tau_{\text {depletion }} \sim M\left(\mathrm{H}_{2}\right) / \mathrm{SFR} \sim 2.4 \times 10^{9} M_{\odot} / 60 M_{\odot} \mathrm{yr}^{-1} \sim 40 \mathrm{Myr}$.

Since we also have a stellar mass of LBG J213512, we can compare the gas depletion time with the time to form the current stellar mass of the system. At the current SFR,

$\tau_{\text {formation }} \sim M_{\text {stars }} / \mathrm{SFR} \sim 6 \times 10^{9} M_{\odot} / 60 M_{\odot} \mathrm{yr}^{-1} \sim 100 \mathrm{Myr}$,

which is comparable to the assumed age of the stellar population.

\section{DISCUSSION}

Our observations of LBG J213512 demonstrate that it is a relatively massive galaxy hosting an equally massive gas reservoir and has significant on-going star formation. The majority of the baryons in the central regions of the galaxy are in the form of stars. We estimate that the stellar population of the LBG could have been formed at the current SFR within $\sim 100 \mathrm{Myr}$ and that if the current SFR continues, then the cold gas reservoir will be exhausted within $\lesssim 40$ Myr unless it is replenished. Thus, it appears that we are seeing the LBG in the last half of its current star formation episode. We now compare the gas properties of LBG J213512 to other similarly well-studied sources and populations at low and high redshifts.

\subsection{Comparison to Other Populations}

We first compare LBG J213512 to the only other CO-detected LBG: cB58 (Baker et al. 2004). The intrinsic CO line luminosity and gas and dynamical masses we derive for LBG J213512 are $L_{\mathrm{CO}(3-2)}^{\prime}=3.0 \times 10^{9} \mathrm{~K} \mathrm{~km} \mathrm{~s}^{-1} \mathrm{pc}^{2}, M_{\text {gas }}=2.5 \times 10^{9} M_{\odot}$, and $M_{\text {dyn }}(<1 \mathrm{kpc})=8.4 \times 10^{9} \csc ^{2} i M_{\odot}$, with a gas fraction within $1 \mathrm{kpc}$ of $f_{\text {gas }}=0.30 \sin ^{2} i$. Using the same conversion factors for cB58, $\alpha=0.8$ and $r_{32}=1$, we estimate $L_{\mathrm{CO}(3-2)}^{\prime}=0.43 \times$ $10^{9} \mathrm{~K} \mathrm{~km} \mathrm{~s}^{-1} \mathrm{pc}^{2}, M_{\text {gas }}=0.34 \times 10^{9} M_{\odot}$, and $M_{\text {dyn }} \sim 10 \times$ $10^{9} \csc ^{2} i M_{\odot}$ (which has FWHM $\mathrm{CO}=175 \pm 45 \mathrm{~km} \mathrm{~s}^{-1}$ ), with a gas fraction of $f_{\text {gas }}=0.03 \sin ^{2} i$.

We could attempt to reduce the uncertainties due to the unknown inclination and average the properties of LBG J213512 and cB58, deriving a typical dynamical mass of $M_{\text {dyn }} \sim(3.6 \pm$ $0.4) \times 10^{10} M_{\odot}$ and a gas fraction of $f_{\text {gas }} \sim 0.05 \pm 0.05$ (both for $i=30^{\circ}$ ). However, we note that LBG J213512 has roughly 7 times more cold gas than $\mathrm{cB} 58$, demonstrating that there is a significant variation in the gas content of $L^{*}$ LBGs at $z \sim 3$, which argues against blindly averaging their properties. Our estimate of the SFR for LBG J213512 is 3-4 times higher than cB58's value of $24 M_{\odot} \mathrm{yr}^{-1}$ (Baker et al. 2004), although this comparison uses different indicators in the two LBGs and is thus uncertain. These SFRs suggest comparable gas depletion timescales for LBG J213512 and cB58: $\tau_{\text {depletion }} \lesssim 40$ Myr for LBG J213512 and $\tau_{\text {depletion }} \sim 0.34 \times 10^{9} / 24 \sim 15 \mathrm{Myr}$ for cB58. The variations in gas mass, as well as the short depletion timescales, probably reflects the brevity of the star formation events and hence the strong variation within the population in the amount of gas consumed at any time. In terms of the comparison of LBG J213512 and cB58, these appear to be similarly massive $L^{*}$ LBGs, although LBG J213512 is younger, more gas-rich, and forming stars at a higher rate than $\mathrm{cB} 58$.
The only high-redshift galaxy population for which there are large numbers of sources with reliable cold gas masses are submillimeter galaxies (SMGs). Taking the average CO line luminosity and gas mass for cB58 and LBG J213512, we find that the "typical" LBG has an intrinsic line luminosity 20 times lower than the median for SMGs $\left(\mathrm{cf} .\left\langle L_{\mathrm{CO}}^{\prime}\right\rangle=(3.8 \pm 2.3) \times 10^{10}\right.$ and $\left\langle M_{\text {gas }}\right\rangle=(3.0 \pm 1.6) \times 10^{10}$; Greve et al. 2005). However, we note that the SMGs studied by Greve et al. (2005) are not strongly lensed, and hence the sensitivity limits of their survey preclude the detection of sources with $L_{\mathrm{CO}}^{\prime} \lesssim 1 \times 10^{10} \mathrm{~K} \mathrm{~km} \mathrm{~s}^{-1} \mathrm{pc}^{2}$, and that in addition, the typical SMGs lie at somewhat lower redshifts than the LBGs (although those SMGs at $z \sim 3$ appear comparable to the general population). The CO line widths of LBG J213512 and cB58 are 3 times lower than the median of SMGs (Greve et al. 2005), with this emission arising in a region estimated to be half the size of that in SMGs ( Tacconi et al. 2006). The typical gas-todynamical mass fraction in SMGs is estimated to be $\sim 0.3$, assuming a merger model (Greve et al. 2005), while they have SFEs of $L_{\mathrm{FIR}} / M_{\mathrm{H}_{2}} \sim 450 \pm 170 L_{\odot} M_{\odot}^{-1}$ (Greve et al. 2005), gas-to-dust mass ratios of $\sim 200$ (with about factor of a few uncertainty in the dust mass alone), and gas surface densities of $\Sigma_{\text {gas }} \sim 3000 M_{\odot} \mathrm{yr}^{-1}$ $\mathrm{pc}^{2}$ ( Tacconi et al. 2006).

Overall, this comparison suggests that LBGs and SMGs are equally evolved, but that the cold gas reservoir in LBGs resides in a system which is typically a factor of $\sim 5$ times less massive than in SMGs (Swinbank et al. 2004, 2006), that the LBG's cold gas disks have surface densities 4 times lower than SMGs (if both can be well described as disks), and that LBGs appear to be forming stars less efficiently than typical SMGs, by a factor of $\sim 4$. The two populations do appear to have similar fractions of baryons in cold gas and stars: $M_{\text {gas }} / M_{\text {stars }} \sim 0.2,0.4$, and 0.3 for cB58, LBG J213512, and SMGs, respectively.

We can also compare the LBGs to local populations. We first note that our $24 \mu \mathrm{m}$ detection predicts a far-infrared luminosity for LBG J213512 of $L_{\mathrm{FIR}} \sim 3 \times 10^{11} L_{\odot}$. Even with our estimated factor of 3 uncertainty, this indicates that an LBG is likely to have a far-infrared luminosity comparable to local LIRGs, $L_{\text {FIR }} \gtrsim 10^{11} L_{\odot}$.

Locally, $L_{\mathrm{CO}}^{\prime}$ increases with $L_{\mathrm{FIR}}$ for (U)LIRGs, with the Greve et al. (2005) sample of SMGs extending this trend out to the highest far-infrared luminosities $\left(\gtrsim 10^{13} L_{\odot}\right)$. For comparison, in Figure 3 we have plotted LBG J213512 on the $L_{\mathrm{CO}}^{\prime}-L_{\mathrm{FIR}}$ diagram, along with cB58 and SMM J16359+6612 (with a lensing magnification of $22 \times$ and weak submillimeter emission, making it more similar to LBG J213512 than to the SMG population) from Kneib et al. (2005), LIRGs, ULIRGs, and SMGs. LBG J213512, cB58, and SMM J16359+6612 all lie on the local relation within the considerable uncertainties in their far-infrared luminosities, in the same region of the plot that the local LIRGs occupy. This suggests that LBG J213512 is similar to local LIRGs.

The CO line widths (and hence dynamical masses for similar radii and inclination angles) for LIRGs ( $\langle$ FWHM $\rangle \sim 200 \mathrm{~km} \mathrm{~s}^{-1}$; Sanders et al. 1991) are comparable to LBG J213512 and cB58, suggesting that LBGs are simply gas-rich high-redshift analogs of LIRGs, but possessing a marginally higher star formation efficiency than the range typically seen in local LIRGs (140 $L_{\odot} M_{\odot}^{-1}$ compared to $\sim 1-50 L_{\odot} M_{\odot}^{-1}$ for $\alpha=0.8$; Sanders et al. 1991).

\subsection{The Physics of Star Formation in LBGS}

What do our observations tell us about the structure of the gas disk and the mode of star formation in LBGs?

To investigate the physics of star formation within LBGs in more detail, we can first test whether the star formation activity within LBG J213512 is consistent with the global Schmidt law 


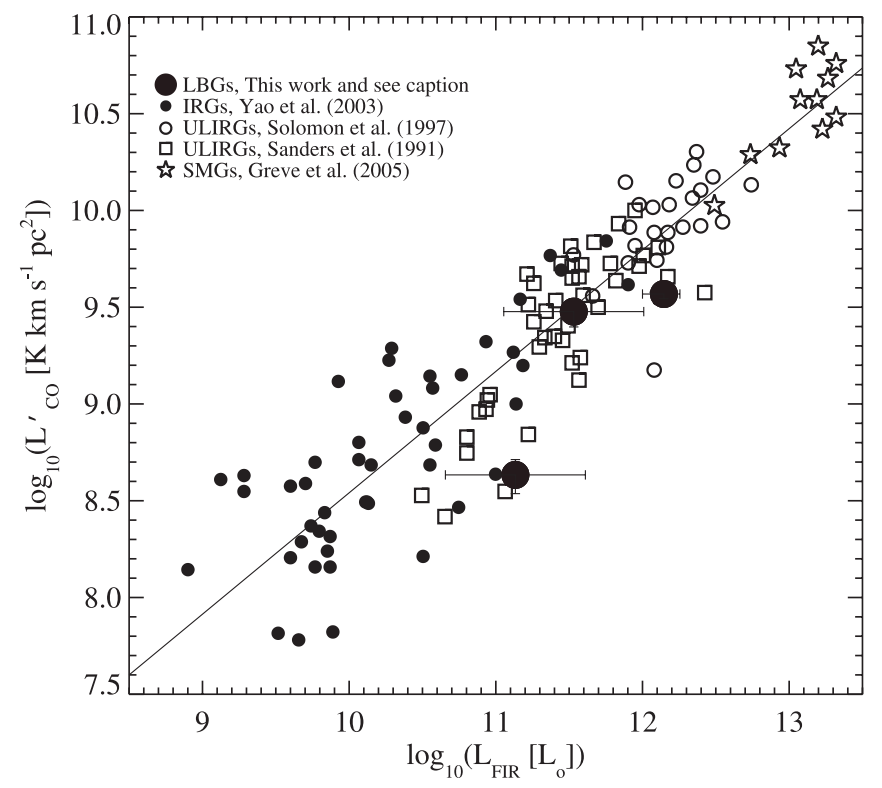

FIG. 3.- Comparison of the CO and far-infrared luminosities for LBGs (including cB58 from Baker et al. 2004 and LBG J213512), and the faint submillimeterselected source SMM J16359+6612 from Kneib et al. (2005) (listed in order of increasing luminosity), LIRGs, ULIRGs, and SMGs. The solid line is the bestfitting relation with a form of $\log L_{\mathrm{CO}}^{\prime}=\alpha \log L_{\mathrm{FIR}}+\beta$ to the LIRGs, ULIRGs, and SMGs from Greve et al. (2005). The LBGs all lie on the relation within their uncertainties and lie in the same region of the plot as the LIRGs. Note that this diagram is a particularly useful diagnostic, since it does not depend on the CO-to- $\mathrm{H}_{2}$ conversion factor.

(Schmidt 1959). Kennicutt (1998) derives a Schmidt law of the form $\Sigma_{\mathrm{SFR}}=A\left(\Sigma_{\mathrm{gas}}\right)^{1.4}$, where $\Sigma_{\text {gas }}$ and $\Sigma_{\mathrm{SFR}}$ are in units of $M_{\odot} \mathrm{yr}^{-1} \mathrm{pc}^{-2}$ and $M_{\odot} \mathrm{yr}^{-1} \mathrm{kpc}^{-2}$, respectively, and $A=(2.5 \pm$ $0.7) \times 10^{-4}$. In deriving this relationship, Kennicutt (1998) assumed $\alpha=4.6$ for all the galaxies (including LIRGs) and noted that the scatter around the relation is large, with individual galaxies lying up to a factor of 7 off the mean relation.

Therefore, we follow Kennicutt (1998) and use $\alpha=4.6$ for the gas surface densities in this calculation. For LBG J213512, we estimate a normalization of $A=1.6_{-1.1}^{+3.2} \times 10^{-4}$, where the large error accounts for the uncertainty in the true SFR. In contrast, cB58 predicts a normalization of $A \sim 9.3 \times 10^{-4}$ with a similar uncertainty. Thus, the two LBGs bracket the local Schmidt law and are each consistent with it within the large scatter and uncertainties.

To determine the likely structure of the gas reservoir, we use the gas mass and assume a radius of $1 \mathrm{kpc}$ to infer a mass surface density of $\Sigma=760 \pm 130 M_{\odot} \mathrm{pc}^{-2}$. If the gas is present in a disk, we can test whether the material will be unstable to bar formation. The Toomre stability criterion states that $Q=\sigma \kappa / 2 \pi G \Sigma \gtrsim 1$ in order for a gaseous disk to be stable (e.g., Binney \& Tremaine 1987), where $\sigma$ and $\kappa$ are the velocity dispersion and the circular frequency of the disk, respectively. For a surface density of $760 M_{\odot} \mathrm{pc}^{-2}, Q<1$ for any realistic value of $\sigma$ and $\kappa$. This suggests that if the gas is present in a disk, it will be unstable to bar formation and will collapse on a timescale of $\tau_{\text {rot }} \sim 60 \mathrm{Myr}$, comparable to the estimated remaining lifetime of the burst.

Following Tacconi et al. (2006), we derive the maximal SFR and SFR surface density for the average LBG with an inclination angle of $i=30^{\circ}$ to be $\sim 20 M_{\odot} \mathrm{yr}^{-1}$ and $\sim 7 M_{\odot} \mathrm{yr}^{-1} \mathrm{kpc}^{-2}$, respectively. These are comparable to the observed average SFR and SFR surface density of $\sim 40 M_{\odot} \mathrm{yr}^{-1}$ and $\sim 7 M_{\odot} \mathrm{yr}^{-1} \mathrm{kpc}^{-2}$, respectively. Hence we conclude that LBGs could be maximal starbursts if our assumptions of the geometry and star formation efficiency are all correct. Our observations appear to point to- ward the star formation in LBG J213512 occurring in an intense central starburst, similar to the activity seen in local LIRGs and ULIRGs. Many of the physical properties we derive for the LBG mirror those of local LIRGs. However, many of these conclusions are sensitive to the conversion factor between the $\mathrm{CO}$ and $\mathrm{H}_{2}$ gas masses, $\alpha$, and so we must re-examine this choice.

The values of $\alpha=0.8 M_{\odot}\left(\mathrm{K} \mathrm{km} \mathrm{s}^{-1} \mathrm{pc}^{2}\right)^{-1}$ and $r_{32}=1$ we adopted are derived from luminous local starburst galaxies in which the molecular gas is distributed in an extensive intercloud medium (Solomon et al. 1997), rather than as discrete giant molecular clouds (GMCs). Thus, lower $\mathrm{CO}$ to $\mathrm{H}_{2}$ ratios in these more energetic environments are potentially an indication of a mode of star formation associated with bulge formation, with higher ratios being more prevalent in quiescently star-forming disks. A value of $\alpha=0.8$ is also adopted for high-redshift submillimeter galaxies (which are believed to be scaled-up highredshift analogs of local ULIRGs; Greve et al. 2005; Tacconi et al. 2006). If we had instead assumed that the cold gas is distributed in stable GMCs, as in our own galaxy, then the predicted gas masses are 9 times higher (taking $\alpha=4.6 M_{\odot}\left[\mathrm{K} \mathrm{km} \mathrm{s}^{-1} \mathrm{pc}^{2}\right]^{-1}$ and $r_{32}=0.65$; Solomon \& Barrett 1991; Mauersberger et al. 1999; Dumke et al. 2001; Yao et al. 2003).

We currently cannot constrain $\alpha$ in high-redshift galaxies directly, and so we must examine whether adopting the Milky Way conversion factors would substantially alter our interpretation of the LBGs (cf. Baker et al. 2004). Adopting $\alpha=4.6$ and $r_{32}=$ 0.65 increases the gas masses for LBG J213512 to $22 \times 10^{9} M_{\odot}$, which is comparable to the cold gas masses derived for typical SMGs (Greve et al. 2005) and exceeds the dynamical mass of the galaxy for inclinations of $i \gtrsim 40^{\circ}$. Similarly, cB58's gas mass increases to $3 \times 10^{9} M_{\odot}$. The star formation efficiency, $L_{\mathrm{FIR}} / M_{\mathrm{H}_{2}}$, for LBG J213512 drops to $\sim 15 L_{\odot} M_{\odot}^{-1}$, while the surface density of the gas disk increases dramatically to $\Sigma_{\text {gas }} \sim 8000 M_{\odot} \mathrm{pc}^{-2}$, larger than the values claimed for SMGs (Tacconi et al. 2006).

It is difficult to understand this mix of properties, with immense gas reservoirs in highly unstable, dense gas disks which are in some respects more extreme than SMGs, but yet result in star formation occurring with an efficiency at least an order of magnitude below that in SMGs. We therefore conclude that it is hard to reconcile the gas properties of LBGs if the CO-to- $\mathrm{H}_{2}$ conversion factor is similar to the Milky Way. Instead, we suggest that the high gas surface density and low star formation efficiency are most naturally explained if the gas is distributed in a similar manner to local ULIRGs and SMGs, and the dominant star formation mode follows that seen in local luminous starbursts (e.g., ULIRGs).

However, the cost of adopting $\alpha=0.8$ is that the gas fraction for cB58 drops to just $f_{\text {gas }} \sim 0.03 \sin ^{2} i$, which seems uncomfortably low for a young high-redshift galaxy which is still forming stars. One potential solution is to adopt different $\mathrm{CO}-$ to- $\mathrm{H}_{2}$ conversion factors in the two LBGs, with the more active LBG J213512 having a lower value than $\mathrm{cB} 58$. This could reflect a period of bulge formation in LBG J213512, while cB58 is currently in a less active phase of star formation. Such a complication is of course unjustified given the current information we have, and we must instead look forward to observations of cold gas in a larger number of LBGs to allow us to place limits on $\alpha$ using the same dynamical arguments employed by Solomon et al. (1997).

\section{CONCLUSIONS}

We have carried out millimeter interferometry and mid-infrared imaging of a strongly lensed LBG at $z=3.07$. We detect strong $\mathrm{CO}(3-2)$ emission with a line width of $190 \pm 24 \mathrm{~km} \mathrm{~s}^{-1}$. Although the lensing magnification of the entire UV source is $\sim 28 \times$, the position of the $\mathrm{CO}$ emission appears coincident with 
a UV component in the source plane which has a magnification of $\sim 8 \times$. Correcting for the magnification of the CO source, LBG $\mathrm{J} 213512$ has an inferred gas mass of $(2.4 \pm 0.4) \times 10^{9} M_{\odot}$ and a dynamical mass of $(8.4 \pm 1.4) \times 10^{9} \csc ^{2} i M_{\odot}$, within an estimated radius of $\sim 1 \mathrm{kpc}$. Fitting to the observed $g, R_{606}, K$, and our Spitzer IRAC 3.6-8.0 $\mu \mathrm{m}$ photometry, we derive a stellar mass of $\sim(6 \pm 2) \times 10^{9} M_{\odot}$. We also use Spitzer/MIPS $24 \mu \mathrm{m}$ imaging to estimate a far-infrared luminosity of $\sim 3 \times 10^{11} L_{\odot}$ with an uncertainty of a factor of 3 . From this we derive a star formation rate of $\sim 60 M_{\odot} \mathrm{yr}^{-1}$ and hence a star formation efficiency of $\sim 140 L_{\odot} M_{\odot}^{-1}$. Based on this star formation rate, we estimate that the current activity in LBG J213512 could have formed the current stellar mass in a period of $\sim 100 \mathrm{Myr}$ and can continue for a further $\sim 40 \mathrm{Myr}$ before the gas reservoir is exhausted.

We find that the CO line luminosity and inferred gas mass for LBG J213512 are $\sim 7$ times higher than that measured for the only other CO-detected LBG, cB58, indicating a significant variation in the gas content of $L^{\star}$ LBGs at $z \sim 3$. In contrast, the two LBGs have effectively identical CO line widths, indicating similar dynamical masses (within the uncertainties of their unknown inclinations). Thus there is a large range in gas-to-dynamical mass ratio between $\mathrm{cB} 58$ and LBG J213512, $M_{\text {gas }} / M_{\text {dyn }} \sim 0.03-$ $0.3 \sin ^{2} i$, which may reflect the short timescales for star formation in these galaxies. Following Kennicutt (1998), we use the gas and star formation surface densities to derive the Schmidt law and find that both LBG J213512 and cB58 are consistent with the Schmidt law.

We compare our observations of LBG J213512 to far-infrared luminous galaxy populations at low and high redshifts. We find that the star formation efficiency we derive for LBG J213512 is slightly above the range of values derived for similarly farinfrared luminous local galaxies (LIRGs), but is significantly below the typical efficiency for high-redshift submillimeter galaxies $\left(\sim 450 L_{\odot} M_{\odot}^{-1}\right)$. The gas and dynamical masses we find are also an order of magnitude smaller than estimated for SMGs. We suggest that LBG J213512 has many features in common with local LIRGs and hence that the star formation activity in this highredshift system may be closely related to the mode of star formation seen in these galaxies.

A major uncertainty in our analysis is the choice of the CO-to$\mathrm{H}_{2}$ conversion factor $(\alpha)$ we should adopt. The two most commonly used assumptions, $\alpha=0.8$ and $r_{32}=1$, appropriate for local ULIRGs, or $\alpha=4.6$ and $r_{32}=0.65$, as measured for the
Milky Way, produce a factor of $\sim 9$ variation in the expected gas masses and all the associated derived properties. We argue that selecting an $\alpha$ and $r_{32}$ similar to comparably far-infrared luminous galaxies at the present day yields a system with more easily understood properties than the more gas-rich system predicted by $\alpha=4.6$ and $r_{32}=1$. However, it is clear that a measurement of other CO transitions for LBG J213512, accessible from the GBT or from the PdB, would place a constraint on the temperature and density of the molecular gas (e.g., Hainline et al. 2006) and allow a more accurate determination of the line luminosity ratio, $r_{32}$, and hence the total gas mass of the system. Placing limits on the CO-to- $\mathrm{H}_{2}$ conversion factor, $\alpha$, in LBGs will require either high angular resolution CO mapping (to resolve the gas disks and solve for their inclination), which could be within the grasp of the most extended IRAM configurations, or from a statistical study of the gas-to-dynamical mass ratios for a large sample of LBGs with CO detections. The latter may have to wait until the advent of ALMA.

Overall, these observations effectively harness a gravitational lens to boost the light grasp of the IRAM and Spitzer telescopes by a factor of up to $30 \times$, while improving the effective resolution to $\sim 0.2^{\prime \prime}$. The next step is to observe the system at higher resolution with IRAM (FWHM $\sim 0.3^{\prime \prime}$ ) in order to dissect the gas distribution on the smallest scales of $\sim 0.02^{\prime \prime}$ (corresponding to $\sim 0.2 \mathrm{kpc}$ in the image plane), providing a preview of the capabilities of ALMA. Such observations will allow us to address questions which are drivers of the ALMA, SKA, and JWST science cases, yielding insights into the kinematics of the interstellar medium in a normal, young galaxy seen 12 billion years ago.

This work is based on observations carried out with the IRAM PdBI. IRAM is supported by INSU/CNRS (France), MPG (Germany), and IGN (Spain). This work also makes use of data obtained with Spitzer, which is operated by the Jet Propulsion Laboratory, California Institute of Technology, under a contract with NASA. We thank the Spitzer director, Tom Soifer, for the award of the Spitzer DDT. We thank Max Pettini, Alice Shapley, and Dave Alexander for useful discussions and help, and an anonymous referee for a constructive report. K. E. K. C., A. M. S., and J. E. G. acknowledge support from PPARC. I. R. S. acknowledges support from the Royal Society.

Baker, A. J., Tacconi, L. J., Genzel, R., Lehnert, D., \& Lutz, D. 2004, ApJ, 604,

\section{REFERENCES}

125

Baugh, C. M., Lacey, C. G., Frenk, C. S., Granato, G. L., Silva, L., Bressan, A., Benson, A. J., \& Cole, S. 2005, MNRAS, 356, 1191

Bell, E. F., et al. 2005, ApJ, 625, 23

Binney, J., \& Tremaine, S. 1987, Galactic Dynamics (Princeton: Princeton Univ. Press)

Blain, A. W., Smail, I., Ivison, R. J., Kneib, J.-P., \& Frayer, D. T. 2002, Phys. Rep., 369, 111

Bolzonella, M., Miralles, J.-M., \& Pello, R. 2000, A\&A, 363, 476

Borys, C., Smail, I., Chapman, S. C., Blain, A. W., Alexander, D. M., \& Ivison, R. J. 2005, ApJ, 635, 853

Bruzual, G., \& Charlot, S. 1993, ApJ, 405, 538

Calzetti, D., Armus, L., Bohlin, R. C., Kinney, A. L., Koornneef, J., \& StorchiBergmann, T. 2000, ApJ, 533, 682

Carilli, C. L., \& Wang, R. 2006, AJ, 132, 2231

Chapman, S. C., et al. 2000, MNRAS, 319, 318

Dale, D. A., \& Helou, G. 2002, ApJ, 576, 159

Dale, D. A., Helou, G., Contursi, A., Silbermann, N. A., \& Kolhatkar, S. 2001, ApJ, 549, 215

Dumke, K., Nieten, C., Thuma, R., Wielebinski, R., \& Walsh, W. 2001, A\&A, 373,853

Dye, S., Smail, I., Swinbank, A. M., Ebeling, H., \& Edge, A. C. 2007, MNRAS, in press (arXiv: 0705.1720v1)

Geach, J. E., et al. 2006, ApJ, 649, 661

Greve, T. R., et al. 2005, MNRAS, 359, 1165

Guilloteau, S., et al. 1992, A\&A, 262, 624

Hainline, L. J., Blain, A. W., Greve, T. R., Chapman, S. C., Smail, I., \& Ivison,

R. J. 2006, ApJ, 650, 614

Kennicutt, R. C., Jr. 1998, ApJ, 498, 541

Kneib, J.-P., Neri, R., Smail, I., Blain, A., Sheth, K., van der Werf, P., \& Knudsen, K. K. 2005, A\&A, 434, 819

Law, D. L., Steidel, C. C., Erb, D. K., Pettini, M., Reddy, N. A., Shapley, A. E., Adelberger, K. L., \& Simenc, D. J. 2007, ApJ, 656, 1

Leitherer, C., et al. 1999, ApJS, 123, 3

Mauersberger, R., Henkel, C., Walsh, W., \& Schulz, A. 1999, A\&A, 341, 256

Pettini, M., Rix, S. A., Steidel, C. C., Adelberger, K. L., Hunt, M. P., \& Shapley, A. E. 2002, ApJ, 569, 742

Pettini, M., Steidel, C. C., Adelberger, K. L., Dickinson, M., \& Giavalisco, M. 2000, ApJ, 528, 96

Reddy, N. A., \& Steidel, C. C. 2004, ApJ, 603, L13

Reddy, N. A., Steidel, C. C., Fadda, D., Yan, L., Pettini, M., Shapley, A. E.,

Erb, D. K., \& Adelberger, K. L. 2006, ApJ, 644, 792

Sanders, D. B., Scoville, N. Z., \& Soifer, B. T. 1991, ApJ, 370, 158 
Schmidt, M. 1959, ApJ, 129, 243

Seaquist, E., Yao, L., Dunne, L., \& Cameron, H. 2004, MNRAS, 349, 1428

Shapley, A. E., Steidel, C. C., Adelberger, K. L., Dickinson, M., Giavalisco, M., \& Pettini, M. 2001, ApJ, 562, 95

Shapley, A. E., Steidel, C. C., Erb, D., Reddy, N. A., Adelberger, K. L., Pettini, M., Barmby, P., \& Huang, J. 2005, ApJ, 626, 698

Shapley, A. E., Steidel, C. C., Pettini, M., \& Adelberger, K. L. 2003, ApJ, 588, 65

Smail, I., et al. 2007, ApJ, 654, L33

Solomon, P. M., \& Barrett, J. W. 1991, in IAU Symp. 146, Dynamics of Galaxies and Their Molecular Cloud Distributions, ed. F. Combes \& F. Casoli (Dordrecht: Kluwer), 235

Solomon, P. M., Downes, D., Radford, S. J. E., \& Barrett, J. W. 1997, ApJ, 478, 144
Solomon, P. M., \& Vanden Bout, P. A. 2005, ARA\&A, 43, 677

Somerville, R. S., Primack, J. R., \& Faber, S. M. 2001, MNRAS, 320, 504

Spergel, D. N., et al. 2003, ApJS, 148, 175

Steidel, C. C., Giavalisco, M., Pettini, M., Dickinson, M., \& Adelberger, K. L. 1996, ApJ, 462, L17

Swinbank, A. M., Chapman, S. C., Smail, I., Lindner, C., Borys, C., Blain, A. W., Ivison, R. J., \& Lewis, G. F. 2006, MNRAS, 371, 465

Swinbank, A. M., Smail, I., Chapman, S. C., Blain, A. W., Ivison, R. J., \& Keel, W. C. 2004, ApJ, 617, 64

Tacconi, L. J., et al. 2006, ApJ, 640, 228

Wall, J. V., \& Jenkins, C. R. 2003, Practical Statistics for Astronomers (Cambridge: Cambridge Univ. Press)

Yao, L., Seaquist, E. R., Kuno, N., \& Dunne, L. 2003, ApJ, 588, 771

Yee, H. K. C., Ellingson, E., \& Carlberg, R. G. 1996, ApJS, 102, 269 